\title{
Worry and anger rumination in fibromyalgia syndrome
}

\author{
A. Ricci ${ }^{1}$, S. Bonini ${ }^{1}$, M. Continanza ${ }^{1}$, M.T. Turano ${ }^{2}$, E.M. Puliti ${ }^{1,3}$, \\ A. Finocchietti ${ }^{1,4}$, D. Bertolucci ${ }^{5}$ \\ ${ }^{1}$ Scuola Cognitiva, Firenze; ${ }^{2}$ Centro Cognitivismo Clinico, Firenze; ${ }^{3}$ Studi Cognitivi, Milano; \\ ${ }^{4}$ Department of Neuroscience, Psychology, Drug Research and Child's Health, University of Florence; \\ ${ }^{5}$ Versilia Hospital, Division of General Medicine, Rheumatology Section, Lido di Camaiore (LU), Italy
}

\begin{abstract}
SUMMARY
The aim of the present study was twofold: 1) to investigate the psychological profile of patients with fibromyalgia syndrome (FS) as compared to patients with other chronic pain syndromes (CP) and healthy subjects (HS); 2) to examine the associations between anxiety, depression, worry and angry rumination in FS patients. FS patients $(\mathrm{N}=30), \mathrm{CP}$ patients $(\mathrm{N}=30)$ and $\mathrm{HS}(\mathrm{N}=30)$ completed measurements of anxiety, depression, worry and angry rumination.

FS patients showed higher levels of state and trait anxiety, worry and angry rumination than CP patients and HS, and higher levels of depression than HS. Worry and angry rumination were strongly associated in the FS group. FS patients may use worry and rumination as coping strategies to deal with their negative emotional experience, which might impair their emotional wellbeing. Findings from the present study add to our understanding of the psychological profile of FS patients, and have important implications for developing a tailored CBT protocol for pain management in FS patients.
\end{abstract}

Key words: Fibromyalgia Syndrome, Chronic Pain, Cognitive Variables, Cbt.

Reumatismo, 2016; 68 (4): 195-198

\section{INTRODUCTION}

$\checkmark 1$ ibromyalgia syndrome (FS) is a rheumatic disorder characterized by chronic musculoskeletal pain throughout the body and fatigability, with no abnormal results on routine laboratory tests. The diagnosis is made on the basis of the criteria of the American College of Rheumatology (ACR). These criteria include, additionally: pain upon digital palpation in at least 11 out of 18 tender points (1) and a variety of extra-skeletal symptoms, including sleep disorders, asthenia, cognitive alterations, gastrointestinal problems, anxiety, depression, migraine, paresthesia, etc. (2).

The most widely used framework for explaining and understanding FS is the biopsychosocial model (3). According to this model, the pain experience is the result of a complex interaction of biological, environmental, social, cultural, racial, cognitive, and behavioral variables (4). Adopting the biopsychosocial model, a number of studies have focused on the psychological factors involved in the onset and development of FS. Findings indicate that FS patients, compared with the general population, have higher levels of anxiety and depression and increased comorbidity with mood disorders (5), which are likely to maintain and augment FS symptoms, thus lowering the quality of life (6). Furthermore, in a previous study of ours (7), FS patients were shown to have a different psychological profile from healthy subjects, with higher worry and need for control.

Based on evidence from the literature and our clinical interest, in the present study we focused on the psychological variables of anxiety, depression, worry and angry rumination which are involved in FS. By definition, worry and rumination are cognitive processes characterized by thinking in an abstract and repetitive fashion, with the focusing of attention on negative feelings and thoughts, and their consequences. In par-
Corresponding author

Daniela Bertolucci

Versilia Hospital

Lido di Camaiore (LU), Italy

E-mail: d.bertolucci@usl12.toscana.it 
Table I - Mean (M) scores and standard deviation (SD) obtained at the psychological testing.

\begin{tabular}{|l|c|c|c|c|c|}
\hline & $\begin{array}{c}\text { STAI - Y1 } \\
\text { M (and SD) }\end{array}$ & $\begin{array}{c}\text { STAI - Y2 } \\
\text { M (and SD) }\end{array}$ & $\begin{array}{c}\text { PSWQ } \\
\text { M (and SD) }\end{array}$ & $\begin{array}{c}\text { ARS } \\
\text { M (and SD) }\end{array}$ & $\begin{array}{c}\text { BDIII } \\
\text { M (and SD) }\end{array}$ \\
\hline FS & $59.3(13.8)$ & $51(13.9)$ & $59.5(16.6)$ & $45.7(11)$ & $19.7(3)$ \\
\hline CP & $33.5(11)$ & $26.8(10.1)$ & $32.3(6.5)$ & $25.2(3.4)$ & $31(4.3)$ \\
\hline HS & $28.5(8.9)$ & $25.7(8.1)$ & $30.4(4.5)$ & $25.30(6.9)$ & $0.9(1.0)$ \\
\hline
\end{tabular}

STAI Y1 - Y2: State (Y1) - Trait (Y2) Anxiety Inventory-Form; PSWQ: Penn State Worry Questionnaire; ARS: Anger Rumination Scale; BDI-I: Beck Depression Inventory. FS: individuals with fibromyalgia; CP: individuals with chronic pain syndromes, HS: healthy individuals.

ticular, worry involves thoughts about the future (8) and is activated to anticipate or prevent events with an uncertain outcome, while rumination includes a greater focus on one's negative feelings, in order to understand their causes (9).

In an attempt to describe the cognitive profile of FS patients and suggest directions for cognitive-behavioral therapy to use with these patients, the present study compared FS patients, patients with other chronic pain syndromes $(\mathrm{CP})$, and healthy subjects (HS) in relation to the above-mentioned psychological variables. Furthermore, the associations between these variables were investigated in the FS group.

\section{MATERIALS AND METHODS}

\section{Participants}

Thirty patients ( 27 women, mean age $54 \pm 12$ years) with fibromyalgia diagnosed according to the 1990 ACR criteria $(1,2), 30$ patients ( 22 women, mean age $59 \pm 13$ years) with $\mathrm{CP}$, specifically osteoporosis and osteoarthritis, and $30 \mathrm{HS}$ (matched for age and gender to the patient groups) participated in the study.

Osteoporosis patients presented localized chronic pain in the back or lumbar spine from vertebral fracture (defined with the Genant semi-quantitative method); osteoarthritis patients presented localized chronic pain in affected areas. All participants were recruited in the cities of Viareggio and Forte dei Marmi (Italy), between April and July 2013. Both the FS and CP groups were recruited from the Operative Unit of Rheumatology of USL 12 Versilia. All participants gave their informed consent to participation in the study.

\section{Measurements and procedure}

Participants completed an individually administered battery of self-report measurements including:

1. Socio-demographic information form.

2. State-Trait Anxiety Inventory-Form Y (STAI) (10) to assess anxiety. The Italian version (11) was used in this study.

3. Penn State Worry Questionnaire (PSWQ) (12) to obtain a trait assessment of pathological worry. The PSWQ consists of 16 items. The Italian version (13) was used in this study.

4. Beck Depression Inventory (BDI-I) (14) to assess the severity of depression in adults and adolescents aged 13 and above. The BDI-I includes 21 items. The Italian version by Scilligo (15) was used.

5. Anger Rumination Scale (ARS) (16) to assess the tendency to ruminate about anger, including thoughts of revenge, angry memories, and understanding of causes. The ARS consists of 14 items. The Italian version was used in the present study.

\section{RESULTS}

Table I reports mean scores and standard deviations obtained by the participants of each group at the psychological testing.

A series of one-way analyses of variance (ANOVA) were conducted. Significant between-group differences were found in state anxiety $(\mathrm{F}=62.5 ; \mathrm{p}<0.001)$, trait anxiety $(\mathrm{F}=51.7 ; \mathrm{p}<0.001)$, depression $(\mathrm{F}=707.2 ; \mathrm{p}<0.001)$, worry $(\mathrm{F}=69.9$; $\mathrm{p}<0.001)$, and angry rumination $(\mathrm{F}=66.5$; $\mathrm{p}<0.001)$ scores. Post-hoc multiple comparisons showed that FS patients had significantly higher levels of trait and state 
anxiety, worry, and angry rumination than both CP patients and HS $(\mathrm{p}<0.001)$. FS and $\mathrm{CP}$ patients reported significantly higher depression scores than HS $(p<0.001)$; in addition $\mathrm{CP}$ presented higher depression scores compared to FS $(\mathrm{p}<0.001)$. Figure 1 showed the results.

Spearman's correlation coefficients between study variables were calculated in each group. State and trait anxiety were moderately correlated in the $\mathrm{CP}$ group $(\mathrm{r}=0.41, \mathrm{p}=0.03)$, and strongly associated in the FS group $(\mathrm{r}=0.58, \mathrm{p}=0.001)$. In the FS group only, worry and angry rumination were strongly correlated with both state $(\mathrm{r}=0.59, \mathrm{p}=0.01$, and $\mathrm{r}=0.55, \mathrm{p}=0.002$, for worry and angry rumination, respectively) and trait anxiety $(\mathrm{r}=0.56, \mathrm{p}=0.01$, and $\mathrm{r}=0.71, \mathrm{p}<0.001$, for worry and angry rumination, respectively). Worry was also significantly, if moderately, correlated with depression ( $\mathrm{r}=-0.37, \mathrm{p}=0.04)$. Results of the correlational analysis for the FS group are shown in Table II.

\section{DISCUSSION AND CONCLUSIONS}

Fibromyalgia is a syndrome characterized by chronic, diffuse musculoskeletal pain, and by numerous extra-skeletal manifestations. The psychological factors involved in the onset and evolution of this syndrome have been the object of growing interest in recent years (4). In accordance with the literature and with a previous study of ours (7), findings from the present study suggest that FS patients have a different psychological profile compared to patients with other chronic pain conditions and to healthy indi-

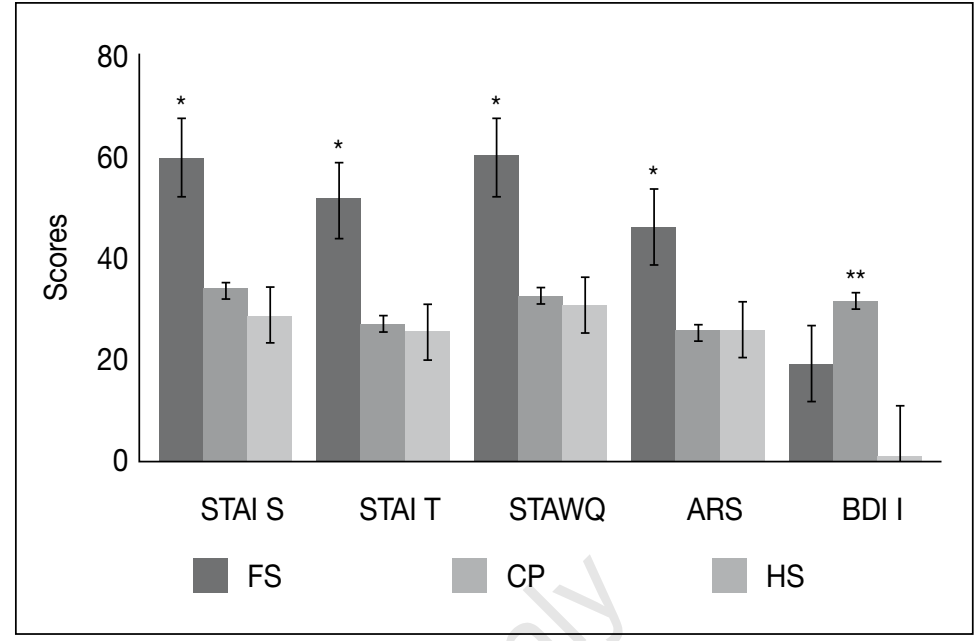

Figure 1 - The scores for the STAI S (Y1), STAI T (Y2), PSWQ, ARS, and $\mathrm{BDI} I$ are showed and contrasted for FS, CP, and HS individuals.

viduals. Indeed, FS patients showed higher levels of anxiety than both $\mathrm{CP}$ patients and HS, and reported higher depression than HS. It is noteworthy that, while FS and $\mathrm{CP}$ showed higher depression than HS, the $\mathrm{CP}$ group also presented higher depression compared to the FS. This interesting finding deserves further investigation. A novel finding of the present study was that FS patients showed higher levels of worry and angry rumination than both $\mathrm{CP}$ patients and HS. While higher levels of worry in FS patients compared to healthy controls had been also found in our previous study (7), findings on angry rumination are novel. To the best of our knowledge, this was the first study to investigate angry rumination in FS.

Altogether, findings from this study seem to suggest that FS patients may use worry and rumination as dysfunctional strategies to cope with negative emotions, with con-

Table II - Correlational analysis in fibromyalgia syndrome.

\begin{tabular}{|l|c|c|c|c|c|}
\hline & STAI - Y1 & STAI - Y2 & PSWQ & ARS & BDI-I \\
\hline STAI - Y1 & 1 & 0.41 & 0.59 & 0.55 & \\
\hline STAI - Y2 & & 1 & 0.56 & 0.71 & \\
\hline PSWQ & & & 1 & & -0.37 \\
\hline ARS & & & & 1 & \\
\hline BDI-I & & & & & 1 \\
\hline
\end{tabular}

STAI Y1 - Y2: State (Y1) - Trait (Y2) Anxiety Inventory-Form; PSWQ: Penn State Worry Questionnaire; ARS: Anger Rumination Scale; BDI-I: Beck Depression Inventory. Only significant results are reported $(p<0.05)$. 
sequent negative effects on self-regulation and emotional well-being.

We wish to emphasize that our findings are preliminary and need replication. Replications of these findings would add to our understanding of the psychological profile of patients with chronic rheumatic pain syndromes, and might have important implications for developing a tailored CBT protocol for pain management in FS patients. This might include different treatment modules focused on anxiety, worry, and angry rumination, and aim at an improvement of the patient's ability to cope with his or her painful emotional experiences. Even if literature on fibromyalgia indicates no specific interventions for the treatment of worry and angry rumination, the relatively new approach of metacognitive therapy (MCT, 17) could be used successfully. This approach, initially developed to treat general anxiety and depression (18), has been reported to have effective outcomes also for worry and angry rumination $(19,20)$. In particular, patients are helped to develop new ways for controlling attention and for relating to negative thoughts and beliefs by modifying their metacognitive beliefs, which give rise to unhelpful thinking patterns. In addition, our research group is working on CBT group-therapy interventions for worry and angry rumination in fibromyalgic patients, which seems to have potential outcomes. This very new integrated approach is based on psychoeducation, mindfulness, and problem solving training.

\section{REFERENCES}

1. Wolfe F, Smythe HA, Yunus MB, et al. The American College of Rheumatology 1990 criteria for the classification of fibromyalgia. Arthritis Rheum. 1990; 33: 160-72.

2. Wolfe F, Clauw DJ, Fitzcharles MA, et al. The American College of Rheumatology preliminary diagnostic criteria for fibromyalgia and measurement of symptom severity. Arthritis Care Res 2010; 62: 600-10.

3. Engel GL. The need for a new medical model: a challenge for biomedicine. Science. 1977; 196: 129-36.

4. Cazzola M. Perchè si diventa fibromialgici: ipotesi patogenetiche. In: P. Sarzi-Puttini, Cazzola M, eds. Il manuale del paziente affetto da sindrome fibromialgica. Milano: GPAnet. 2009; 63-77.

5. Alciati A. Aspetti psichiatrici della fibromialgia. In: P. Sarzi-Puttini, Cazzola M, eds. Il manuale del paziente affetto da sindrome fibromialgica. Milano: GPAnet. 2009; 123-129.

6. Gormsen L, Rosenberg R, Bach FW, Jensen TS. Depression, anxiety, health-related quality of life and pain in patients with chronic fibromyalgia and neuropathic pain. Eur J Pain. 2010; 14: 127-38.

7. Bonini S, Continanza M, Rigacci C, et al. Costrutti Cognitivi dell'Ansia e Sindrome Fibromialgica. Psicoter Cognit Comportam. 2014; 20: 219-29.

8. Borkovec TD, Ray WJ, Stöber J. Worry: A cognitive phenomenon intimately linked to affective, physiological, and interpersonal behavioral processes. Cognit Ther Res. 1998; 22: $561-76$

9. Nolen-Hoeksema S, Morrow J, Fredrickson BL. Response styles and the duration of episodes of depressed mood. J Abnorm Psychol. 1993; 102: 20-8.

10. Spielberger CD, Gorsuch RL, Lushene R, et al. Manual for the State-Trait Anxiety Inventory. Palo Alto: Consulting Psychologists Press; 1983.

11. Pedrabissi L, Santinello M. Adattamento italiano S.T.A.I. State-Trait Anxiety Inventory, Forma Y: Manuale. Firenze: Organizzazioni Speciali. 1989.

12. Meyer TJ, Miller ML, Metzger RL, Borkovec TD. Development and validation of the Penn State Worry Questionnaire. Behav Res Ther. 1990; 28: 487-95.

13. Morani S, Pricci D, Sanavio E. "Penn State Worry Questionnaire" and "Worry Domains Questionnaire": Italian version and reliability. Psicoterapia Cognitiva e Comportamentale. 1999; 5: 195.

14. Beck AT, Ward CH, Mendelson M, et al. An inventory for measuring depression. Archiv Gen Psychiatr. 1961; 4: 561-71.

15. Scilligo P. Caratteristiche psicometriche del Questionario di Depressione di Beck. Psicol Clin. 1983; 3: 397-405.

16. Sukhodolsk, DG, Golu, A, Cromwell EN. Development and validation of the anger rumination scale. Personality and Individual Differences 2001; 31: 689-700.

17. Wells A, Matthews G. Attention and emotion: a clinical perspective. Erlbaum: Psychology press. 1994.

18. Wells A. Metacognitive therapy for anxiety and depression. New York: Guilford Press. 2009.

19. Papageorgiou C, Wells A. Depressive rumination: nature, theory and treatment. Chichester, UK: Wiley. 2004.

20. Davey G, Wells A. Worry and its psychological disorders: theory, assessment and treatment. Chichester, UK: Wiley. 2006. 